\title{
Intracranial dural arteriovenous fistulas: a review
}

\begin{abstract}
Intracranial dural arteriovenous fistulas, a seldom-encountered type of vascular malformations, are nonetheless clinically relevant because a misdiagnosis may lead to permanent morbidity and mortality. Modern brain imaging techniques such as CT and MRI angiography have a substantial role in the preoperative work-up, yet digital subtraction angiography remains the neuroimaging mainstay. The most important factor guiding management is the presence of cortical venous reflux, significantly associated with aggressive symptoms. Cutting edge developments in endovascular and neurosurgical treatment of these vascular malformations, which should be undertaken in specialized referral centers, have substantially improved their prognosis.
\end{abstract}

Keywords: Intracranial dural arteriovenous fistulas, Embolisation, Surgery, Multidisciplinary treatment, DAVF, Cognard classification, Borden classification

\author{
Volume I Issue 2 - 2014 \\ Francesco Signorelli, ${ }^{1,2}$ Benjamin Gory, ${ }^{3}$ \\ Rodolfo Maduri, ${ }^{4}$ Jacques Guyotat,' Isabelle \\ Pelissou-Guyotat,' Domenico Chirchiglia, ${ }^{2}$ \\ Roberto Riva, ${ }^{3}$ Francis Turjman ${ }^{3}$ \\ 'Department of Neurosurgery, Hospices Civils de Lyon, France \\ ${ }^{2}$ Department of Experimental and Clinical Medicine, University \\ "Magna Graecia" of Catanzaro, Italy \\ ${ }^{3}$ Department of Neuro Interventional Radiology, Hospices Civils \\ de Lyon, France \\ ${ }^{4}$ Department of Neurosurgery, University Hospital of Vaud, \\ Switzerland

\begin{abstract}
Correspondence: Francesco Signorelli, Department of Neurosurgery and Neurology, Hospices Civils de Lyon, Neurological and Neurosurgical Hospital, 59 Bd Pinel, 69550 Lyon-Bron, France and Department of Experimental and Clinical Medicine, University “Magna Graecia” of Catanzaro,Viale Europa 80100 Germaneto, ITALY,Tel +33-6 08-35-20-03,
\end{abstract} \\ Email signorelli2007@gmail.com
}

Received: May 02, 2014 | Published: June 06, 2014

\begin{abstract}
Abbreviations: AV, arteriovenous; AVM, arteriovenous malformation; CVD, cortical venous drainage; CT, computed tomography; CTA, computed tomography angiography; DSA, digital subtraction angiography; ECA, external carotid artery; FPD, flat panel detector; ICGA, indocyanine green angiography; IDAVFs, intracranial dural arterio venous fistulas; LEA, liquid embolic agent; MRI, magnetic resonance imaging; MRA, magnetic resonance angiography; SS, superficial siderosis; TAE, transarterial embolization; TVE, trans venous embolization
\end{abstract}

\section{Introduction}

Intracranial dural arteriovenous fistulas (IDAVFs) are a relatively rare entity, whose clinical features and prognosis vary according to the location and risk of bleeding. They are anatomically and pathologically distinct from pial and Parenchymal arteriovenous malformations (AVMs), in that they are supplied by Dural arteries without pial involvement and display a direct arteriovenous shunt toward Dural sinuses and/or pachi (dural) or leptomeningeal (pial) veins with no Parenchymal nidus. A number of radiological classifications have been proposed which are intended to aid in their clinical management. Modern imaging techniques may prove useful in their evaluation and follow-up. However, digital subtraction angiography (DSA) remains the gold standard and is necessary to guide multidisciplinary treatment strategy.

\section{IDAVF}

\section{Definition of IDAVF}

IDAVFs are abnormal AV shunts that receive supply through meningeal arteries or pachimeningeal branches of cerebral arteries and are drained directly through a dural sinus or venous channels tributary of a venous sinus or through dilated or non-dilated superficial or deep leptomeningeal (pial) veins. ${ }^{1}$

\section{Epidemiology}

Their incidence and prevalence are unknown, due to lack of prospective epidemiological studies. Some retrospective series find them to be one tenth as frequent as brain AVMs..$^{2-5}$ IDAVFs tend to present later in life than AVMs, thus they are thought to be acquired rather than congenital. ${ }^{1}$

\section{Etiopathogenesis and clinical presentation}

Although generally idiopathic, IDAVFs may be acquired as a consequence of head trauma, venous sinus stenosis or occlusion and head surgery. ${ }^{1}$ The current pathogenic hypothesis is based on the common observation of association between IDAVFs and venous outflow obstruction: increased venous pressure may favor enlargement of physiologic shunts between duralarteries and sinuses or promote neoangiogenesis through regional venous ischemia. The association of IDAVFs further suggests the role of venous thrombosis in the development of IDAVFs with hypercoagulable state due to antithrombin, protein $\mathrm{C}$ and protein $\mathrm{S}$ deficiencies. ${ }^{6}$ Since the first description of DAVFs in the early ' 70 , the clinical picture has generally been correlated with location and angioarchitecture, especially pattern of venous drainage. ${ }^{7}$ Direct communication between Dural arteries and venous sinus or meningeal venous lacunae with ante grade venous drainage increases flow through Dural sinuses or venous channels without venous congestion and commonly produces only benign symptoms such as headaches, pulsatile tinnitus or exophthalmos. However, venous drainage toward the cavernous sinus may engender painful proptosis, multiple cranial nerve deficits and decreased vision. Conversely, a retrograde venous flow through sinuses or cortical veins as well as a direct cortical venous drainage (CVD), signs of passive venous congestion, carry a high risk of aggressive neurological symptoms and of intracranial hemorrhage, which is even greater in case of dilated venous channels. ${ }^{8}$ Myelopathy develops when venous drainage descends toward the consume dullaris, while it is 
generally absent when perimedullary veins are limited to the cervical spinal cord. ${ }^{9}$ In the presence of cortical or perimedullary venous congestion,vasogenic (extracellular) edema develops first into white matter, leading to gradual neurological impairment with epileptic seizures, progressive cognitive impairment, parkinsonism, cerebella symptoms, cranial nerve abnormalities or myelopathy, depending on the site of IAVDF. At this stage, interruption of the fistula may lead to regression of symptoms..$^{10}$ On the other hand, if the cause of venous congestion persists, cytotoxic edema develops and interruption of the fistula at this stage may not result in neurological improvement. ${ }^{11-16}$ This explains the importance of an early diagnosis and treatment of symptomatic IDAVFs. An uncommon although often-unrecognized mechanism of progressive neurological impairment associated with IDAVFs is continuous or recurrent microhemorrhages into subarachnoid spaces resulting in superficial Siderosis. Resultant hemosiderin deposits in the leptomeninges, pial and subpial layers progressively induce neuronal damage and are responsible for SS manifestations. ${ }^{17}$

\section{Classification and natural history (Figures I-7)}

Several classification systems have been developed to describe and guide the treatment of IDAVFs. The most used are Cognard et al. ${ }^{18}$ and Borden et al. ${ }^{19}$ classifications. Both are based on the pattern of venous drainage, which reflects the risk of hemorrhage. According to Cognard's scheme Type I and II IDAVFs drain directly into dural sinus, the difference being the presence in type I of ante grade flow and in type II of retrograde flow into dural sinus (type II a), cortical (pial) veins (type II b) or both (type II $a+b$ ) (Figures 1-4); type III and IV drain directly into cortical (pial) veins, without dural venous drainage, the difference being the presence in type IV of dilated CVD (Figures 5-7); type V drain into perimedullary veins. Similarly to Cognard's, Borden's type I IDAVFs have drainage into a Dural venous sinus or pachimeningeal veins with ante grade flow (Figures $1 \& 2$ ) and type III DAVFs have the same dural drainage with retrograde flow into the sinus and/or cortical venous reflux (Figures $3 \& 4)$, while type III have drainage directly into cortical veins or an isolated segment of dural sinus with cortical venous reflux (Figures 5-7). According to both systems, the presence of ante grade venous drainage without retrograde flow is associated with a benign clinical course. On the other hand, the factors increasing the risk of aggressive behavior are, in order of importance, the presence of retrograde flow into dural sinus and veins, into cortical or deep veins and the presence of a dilated CVD ${ }^{7}$. While Cognard and Borden type I IDAVFs have a benign course with virtually no risk of aggressive symptoms, type II with retrograde flow only into Dural sinuses and pachimeningealveins are associated in about $37 \%$ of cases with aggressive symptoms other than hemorrhage. Cognard's and Borden type II IDAVFs with reflux into cortical veins have a more aggressive course, presenting with intracranial hemorrhage in $10.7^{18}$ to $18 \%$ of cases,${ }^{19}$ with an annual hemorrhage rate of nearly $6 \%$, while other aggressive symptoms are present in almost $43 \%$ of cases. ${ }^{18}$ Both classifications find that the risk of hemorrhage greatly increases in case of direct drainage into cortical veins, although Borden's classification does not make difference between dilated and non dilated CVD, which is the distinctive feature of Cognard's type IV IDAVFs compared to type III. Several authors report higher bleeding risk in case of venous ectasia (i.e. CVD $>5 \mathrm{~mm}$ and 3 times larger than the diameter of draining vein), regardless of the classification scheme used. Actually, Borden's type III without dilated CVD, matching Cognard's type III IDAVFs, present with hemorrhage in $34^{20}$ to $40 \%$ of cases, ${ }^{18}$ with an annual hemorrhage rate of $10 \%$, while $76 \%$ of Borden's type III with dilated CVD, corresponding to
Cognard's type IV IDAVFs, show a hemorrhagic presentation and have a $21 \%$ annual bleeding rate. Annual hemorrhage risk further increases in case of previous hemorrhage, up to $46 \%{ }^{20}$ Aggressive symptoms other than hemorrhage have a similar frequency in IDAVFs with reflux into cortical veins regardless the presence of direct CVD, between 36 and $43 \%$ of cases. Cognard types V IDAVFs with drainage into perimedullary veins are associated in $50 \%$ of cases with myelopathy. ${ }^{18}$ Borden-Cognard type I IDAVFs may rarely modify and develop CVD, in $1.4 \%$ of cases and occasionally display a pial recruitment when enlarging or becoming more diffuse or following partial embolization. ${ }^{20}$

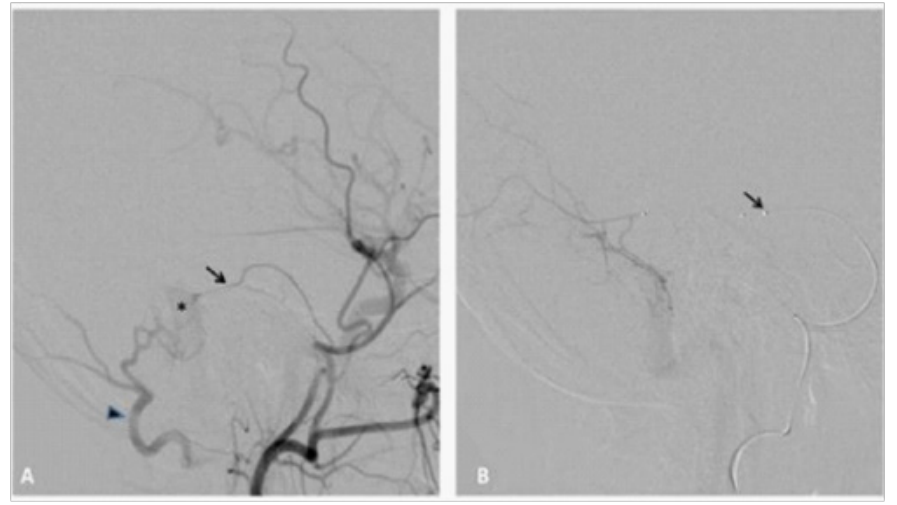

Figure I 50year old man with left pulsatile tinnitus. A. Digital subtraction angiography (DSA) of the of the left external carotid artery (ECA), lateral view, demonstrating a grade I IDAVF of the left lateral sinus supplied by the middle meningeal artery (black arrow) and occipital artery (black arrowhead). Note the early opacification of the lateral sinus (asterisk). Super selective DSA, lateral view, the middle meningeal artery is accessed by a sonic I.2F micro catheter black arrow.
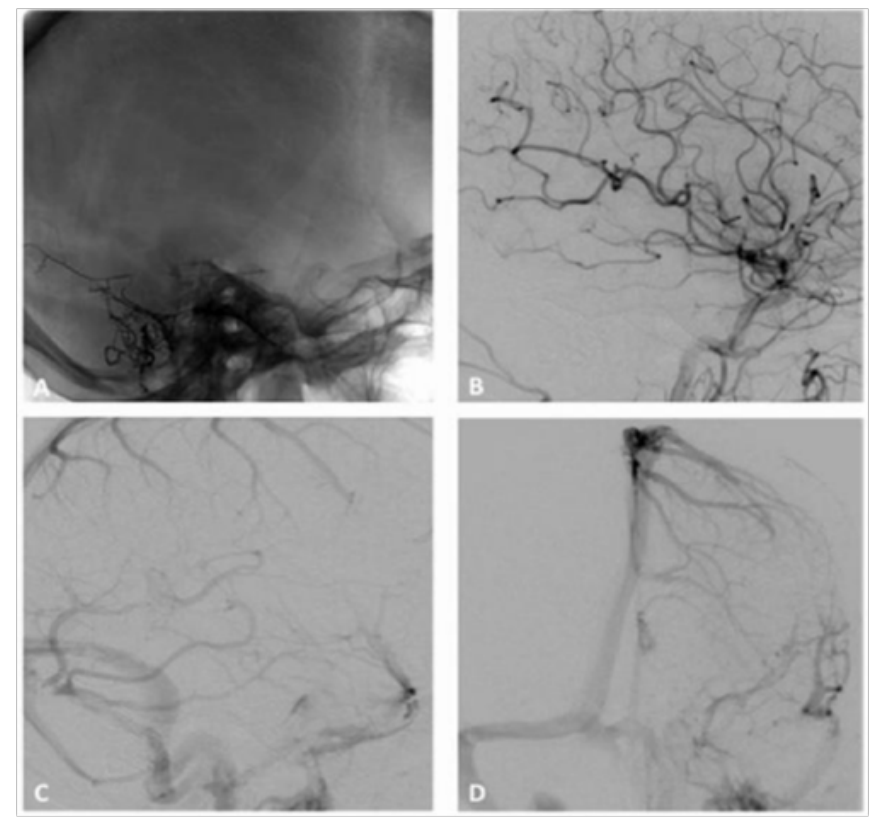

Figure 2 A. Post-embolization radiography, lateral view, showing the cast of Onyx occluding the middle meningeal artery and the occipital artery through an arterioarterial reflux. B. DSA of the common carotid artery, lateral view, showing complete occlusion of the fistula. C and D, DSA of the common carotid artery, lateral and anteroposterior view, showing the patency of the lateral sinus. The patient had resolution of his pulsatile tinnitus, persisting at a 3 years follow-up. 

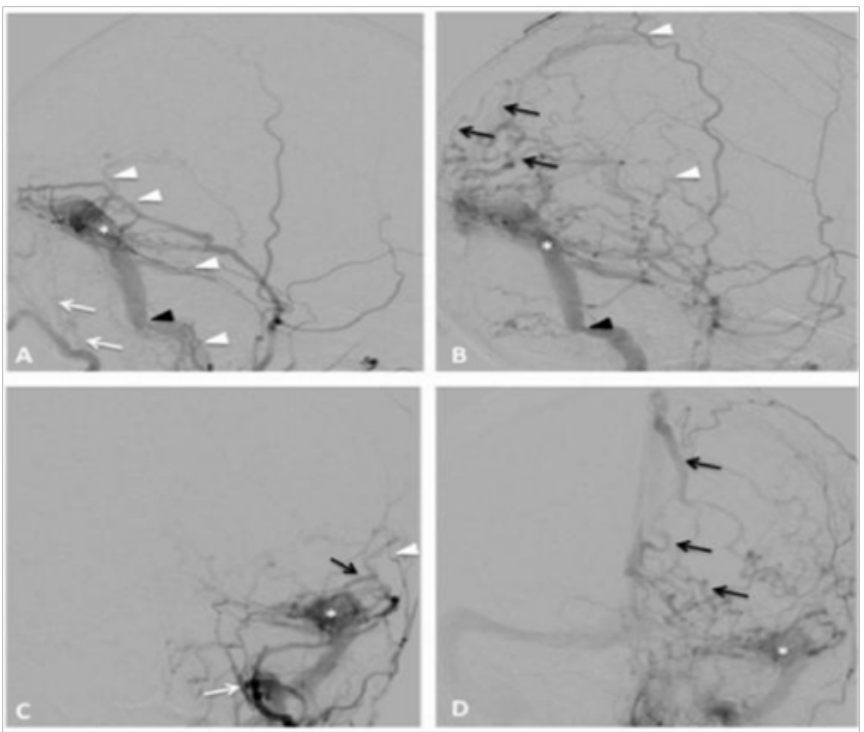

Figure 3 A 43-year-old woman developed a left pulsatile tinnitus. A and B (left ECA injection, lateral projection) $C$ and $D$ (left ECA injection, $A P$ projection) DSA showing a Borden and Cognard grade II IDAVF with multiple shunts between the occipital artery (white arrow), parietal branches of the middle meningeal artery (white arrowheads) and left transverse sinus (asterisk).There is retrograde flow into cortical veins (black arrows). Note the associated sigmoid sinus stenosis (black arrowhead), Just upstream to jugular bulb.
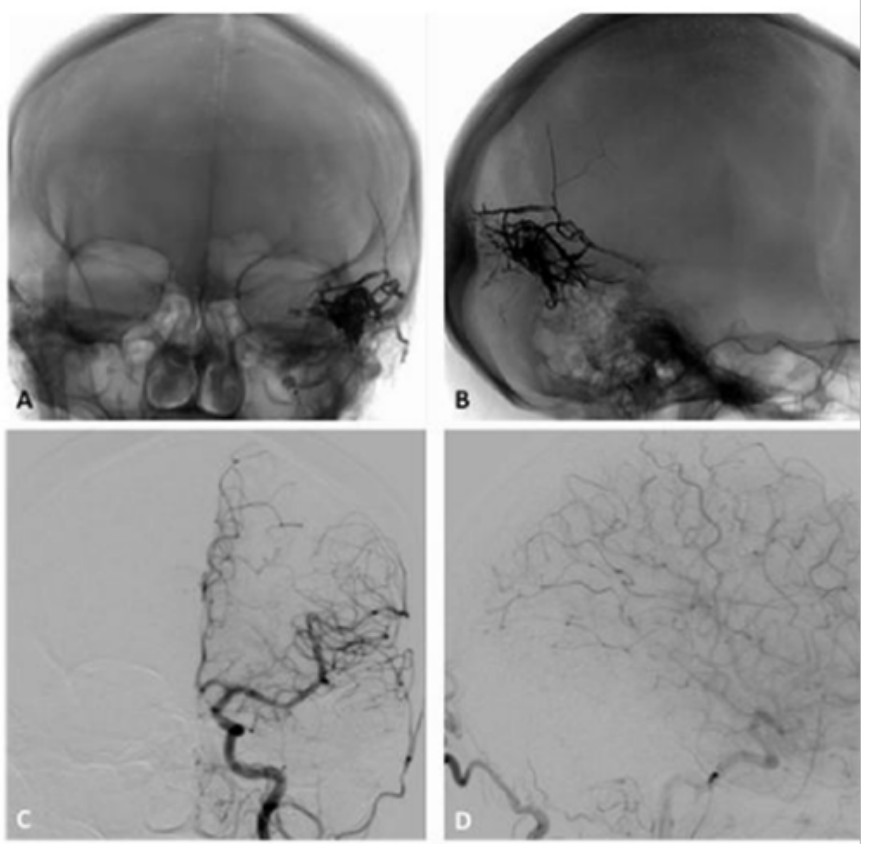

Figure $4 \mathrm{~A}$ (anteroposterior) and $\mathrm{B}$ (lateral), radiography showing the cast of Onyx obliterating the parietal branches of the middle meningeal artery with arterioarterial reflux into homolateral feeders, the cast extending to the arteriovenous junction and the proximal venous outflow. C Anteroposterior and $\mathrm{D}$ lateral projections DSA of the common carotid artery demonstrating a complete obliteration of the fistula.

The initial imaging workup generally includes computed tomography (CT) and magnetic resonance imaging (MRI). In case of cortical venous reflux or direct CVD they generally show direct signs of IDAVFs such as flow voids (due to engorged and tortuouspial or meningeal vessels) and prominent vascular enhancement, in addition to indirect signs such as white matter or basal nuclei hypo(on CT and MRI T1-weighted imaging) and hyper intensity (on MRI T2-weighted imaging) secondary to venous hypertension, as well as hemorrhage and hydrocephalus. ${ }^{21}$ Conversely, in case of type I IDAVFs, conventional imaging is frequently unremarkable ealbeit it can show associated venous sinus stenosis or occlusion. ${ }^{22}$ Given the limitation of CT and MRI, 6-vessels digital subtraction angiography (DSA) is indicated to diagnose and fully evaluate an IDAVF. This should include selective visualization of dural branches from external carotid (ECA), internal carotid (ICA) and vertebral arteries (VA), it should start in the early arterial phase and include late venous phase images. Super selective catheterization of distal feeders allows full delineation of the IDAVF supply and precise location of its nidus, where feeders converge and draining veins originate. It is especially important to identify signs of venous congestion and dilation, the so called "pseudophlebitic pattern", present in $81 \%$ of type II-IV and in only $8 \%$ of type I IDAVFs, that correlates with risk of aggressive symptoms and prompts treatment. ${ }^{22}$ Modern imaging techniques such as CT angiography (CTA), particularly flat panel detector CTA (FPD-CTA), and MRI angiography (MRA) can add high-resolution anatomic details that help outlining arterial feeders, the fistulous site and venous outflow, especially useful for surgical treatment. ${ }^{23,24}$

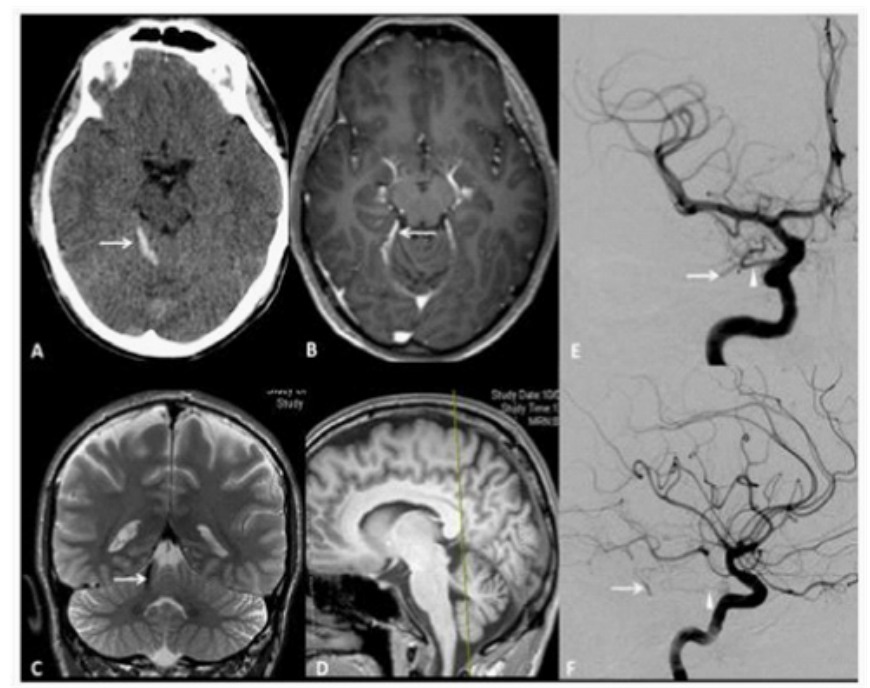

Figure 5 A 33year old man presenting with sudden headache and meningiomes.

A.Axial Head CT scan shows a pialhemorrhage (white Arrow) on the surface of the right anteriorquadrangular lobe of the cerebellum.

B. Axial TI-weighted enhanced MRI showing a small flow void in contact with the right posterior $1 / 3$ of the free tentorialedge, corresponding to a suspended veinbridgingbetween the tentorialconvexity of the cerebellum to a tentorialvenouslacuna.

C. Coronal T2-weighted High resolution image, showing the bridgingvein (white Arrow).

D. Sagittal TI-weighted reference image. The yellow trait corresponds to the level of the coronal cutdepicted in the $\mathrm{C}$ box.

$A P$ and F, LL views of DSA, showing a grade III Borden without venous ectasia, Grade III Cognard IDAVF supplied by the right tentorialartery (Bernasconi and Cassinariartery, white arrowhead), branch of the meningohypophisealtrunk, originatingfrom the intracavernous ICA and drained by the small suspended vein (white arrow) tributary of a tentorialvenouslacunaseen on MRI, whichis the source of hemorrhage. 

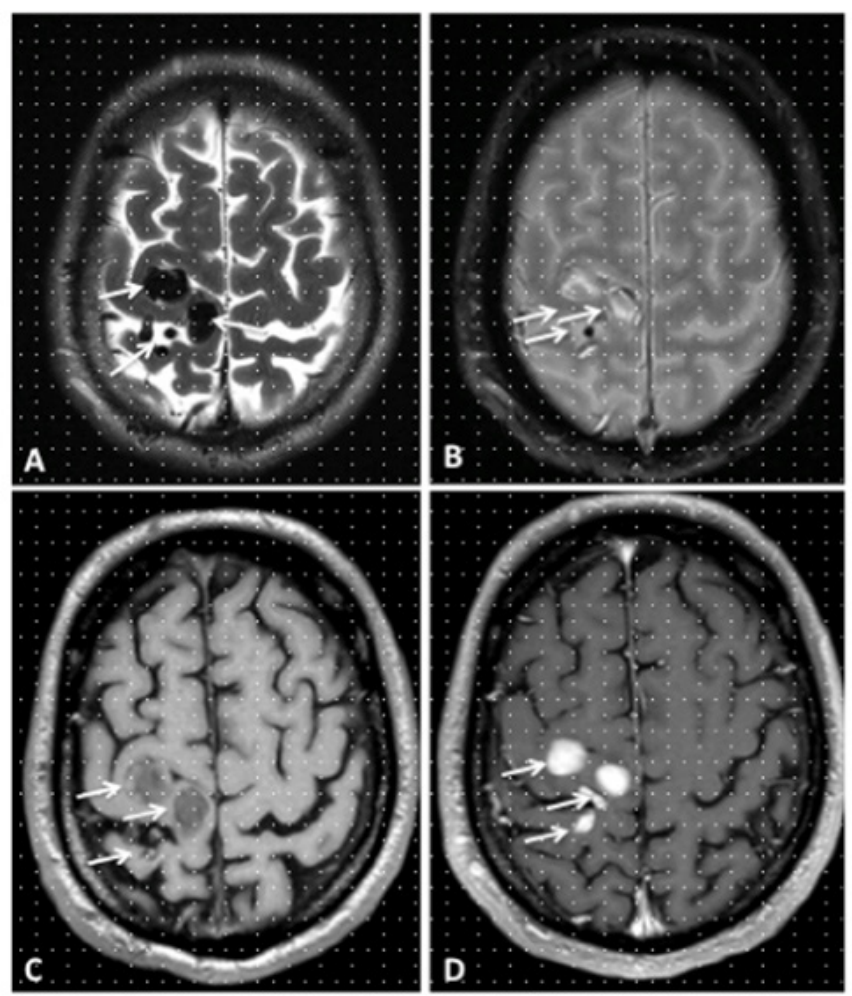

Figure 6 A 50year old man presenting with left partial rolandic seizures.A,T2, $\mathrm{B}, \mathrm{T2}$ * , C, TI-weighted MR sequences before and D, after gadolinium injection, axial view, showing flow voids (white arrows) without haemorrhage in the right rolandic area.
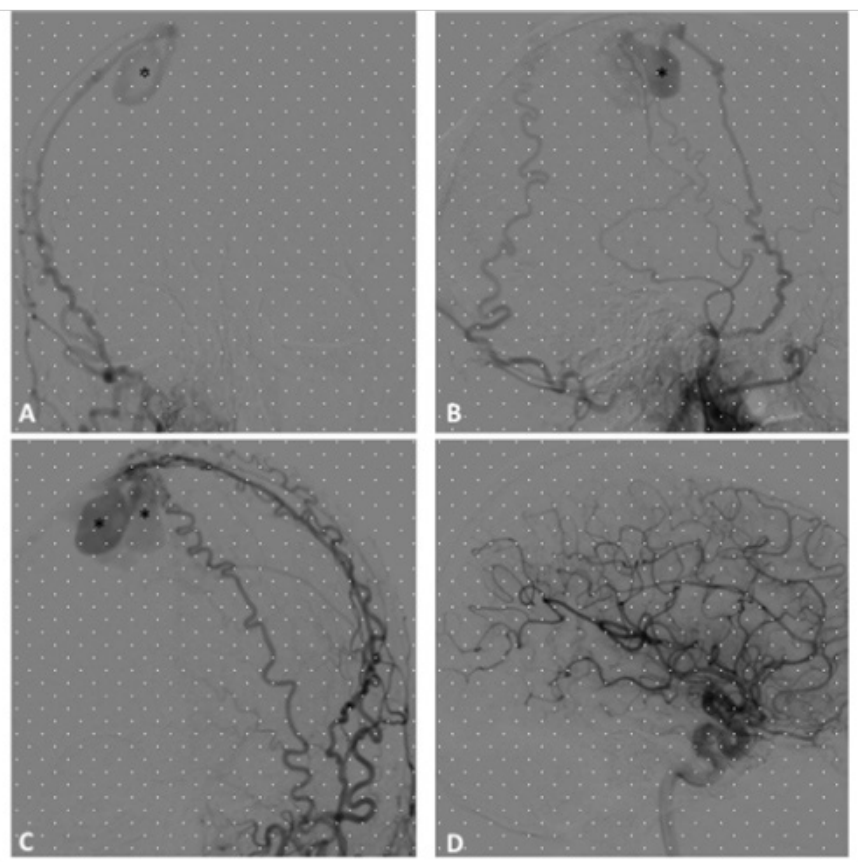

Figure $7 \mathrm{~A}, \mathrm{~B}$, right and $\mathrm{C}$ left external carotid artery injections DSA confirms a Borden grade III IDAVF with venous ectasias (asterisk), which corresponds to Cognard grade IV, with bilateral arterial supply from the middle meningeal and superficial temporal arteries and direct CVD through dilated pial veins tributary of the superior sagittal sinus. D, Lateral projection DSA does not show ICA supply to the fistula.

\section{Treatment}

The expertise of a multidisciplinary staff involving neurosurgeons, interventional Neuroradiologist, neurointensivists and in some cases radiotherapists endorses the best management for these complex lesions and allows correcting weight treatment risks against the expected clinical evolution, especially regarding aggressive neurological symptoms. Cognard and Borden grade I and II IDAVFs without cortical venous reflux and drainage are best managed conservatively, unless annoying symptoms such as pulsatile tinnitus or exophthalmos are long lasting and evaluative. In case of CVD or perimedullary venous drainage with myelopathy, the treatment is warranted soon after diagnosis in order to avoid permanent deficits and prevent the risk of new or repeat hemorrhage. The aim of the treatment is complete interruption of the arteriovenous shunt, incomplete treatment having no protective effect against hemorrhage because fistula will eventually recruit collateral vessels. ${ }^{24}$ However, partial treatment of complex IDAVfs may be a viable option in order to limit neurological morbidity with less operative risks, especially in fragile patients, ${ }^{25,26}$ or to reduce vascularization and simplify subsequent surgical resection. ${ }^{23}$ The current core treatment of IDAVFs is endovascular embolization, which can be achieved through transarterial, transvenously or combined approach. ${ }^{2}$ Transarterial embolization (TAE) is the most employed, favored in case of isolated or severely stenoticdural sinus or when venous outflow channels are tortuous and unviable, unless venous direct puncture is feasible. However, embolization of the sole arterial supply rarely cures IDAVFs because the shunt will ultimately receive supply from newly developed meningeal collaterals..$^{10}$ During TAE the micro catheter should be selectively navigated and wedged as distally as possible inside the feeders and the liquid embolic agent (LEA) injected into the shunt up to occlude the most proximal portion of the draining venous channels. ${ }^{10}$ Both N-butyl-2-cyanoacrylate and Onyx (eV3, Irvine, Ca) can be used. However Onyx, which is non adherent and has less risk of gluing the micro catheter, and has variable viscosity, which improves its penetration into the fistula, is frequently preferred to glue. Its excellent penetration makes possible to treat effectively MultiFinder IDAVFs through a single pedicle. ${ }^{27}$ Another advantage of Onyx is its black color, which allows easier identification of IDAVF during surgery, in case of partial embolization. ${ }^{21}$ Onyx is less suitable to TAE when feeders from ICA or VA are shorter than 15 to $20 \mathrm{~mm}$, because of the risk of stroke due to reflux of this embolic agent into normal vessels, higher than with glue, which polymerizes earlier than Onyx with shorter reflux. ${ }^{24} \mathrm{~A}$ disadvantage of Onyx is its potential toxicity to cranial nerves, which contraindicates its direct injection into arteries supplying them. ${ }^{21}$ Tran venous embolization (TVE) by glue or coils is a good option when arterial feeders are numerous, too tiny and tortuous to be navigated distally and the venous drainage of the IDAVF can be sacrificed without compromising the outflow of normal brain. Moreover, an anatomically isolated dural sinus can be reached transvenously through venous collaterals or even the thrombotic occlusion, which represents an advantage of TVE. However, TVE is not without risks of vessel perforation, venous congestion, leading to brain infarction or hemorrhage and venous outflow modifications, which may engender transient or permanent neurologic deficits. Surgery remains a valid option in case embolization fails, is not feasible or incomplete. Some IDAVFs locations, such as anterior cranial fossa floor, tentorial surface or free edge, are more amenable to surgery because of the difficulty to reach them endovascularly. Likewise, surgery is preferred to embolization for fistulas draining into the superior sagittal or dominant transverse sinus when endovascular treatment would entail 
an undesirable dural sinus sacrifice. ${ }^{23}$ An important landmark for the surgeon is the arterialized vein, following which upstream the nidus can be found. Coagulation of all accessible feeders and disconnection of all draining veins with preservation of normal venous drainage is the aim of surgery. Application of neuronavigation and Intraoperative indocianine green angiography (ICGA) are valuable adjuncts, which allow precisely locating the nidus of the fistula and verifying its complete disconnection from draining veins while preserving normal venous drainage. In case of failure of both endovascular and surgical treatments, stereotactic radio surgery (SRS) represents an alternative. However, this treatment has low success rate as the only treatment ${ }^{28}$, especially in case of complex lesions for which targeting of the shunt is difficult ${ }^{29}$. Moreover, SRS is generally inappropriate for lesions presenting with bleeding because during the latency period of IDAVF obliteration, which lasts several months to a year, the rehemorrhage risk remains elevated ${ }^{10}$.

\section{Conclusion}

IDAVFs are infrequent vascular malformations whose diagnosis and treatment are prerogative of a multidisciplinary team, involving neurosurgeons, interventional Neuroradiologist, neurointensivists and radiotherapists, in highly specialized centers where a multimodality management is offered, thorough clinical evaluation and modern imaging techniques allow to correctly weight treatment risks against the expected clinical evolution, which can be marked by aggressive neurological symptoms. Prognosis of high grade IDAVFs greatly depends on early diagnosis and treatment. Significant advancements in the management of IDAVFs have been made due to the development of recent pre- and Intraoperative imaging methods such as FPD CTA and ICGA, innovative endovascular techniques and introduction of new LEA such as Onyx. However, surgery remains a mainstay in the treatment of IDAVFs that are not amenable to embolization or in conjunction to it.

\section{Acknowledgments}

None.

\section{Conflicts of interest}

The authors declare there are no conflicts of interest related to the article.

\section{References}

1. Awad IA, Little JR, Akarawi WP, Intracranialdural arteriovenous malformations: factorspredisposing to an aggressive neurological course. J Neurosurg. 1990;72(6):839-850.

2. Davies MA, TerBrugge K, Willinsky R, et al. The validity of classification for the clinical presentation of intracranialduralarteriovenousfistulas. $J$ Neurosurg. 1996;85(5):830-837.

3. Davies MA, Ter Brugge K, Willinsky R, et al. The natural history and management of Intracranialdural arteriovenous fistulae, part 2: aggressive lesions. Interv Neuroradiol. 1997;3(4):303-311.

4. Davies MA, Saleh J, Ter Brugge K, et al. The natural history and management of intracranial dural arteriovenous fistulae, part 1: benign lesions. Interv Neuroradiol. 1997;3(4):295-302.

5. Gross BA, Du R. The natural history of cerebral dural arteriovenous fistulae. Neurosurgery. 2012;71(3):594-602.

6. da Costa L, Wallace MC, Ter Brugge KG, et al. The natural history and predictive features of hemorrhage from brain arteriovenous malformations. Stroke. 2009;40(1):100-105.
7. Hernesniemi JA, Dashti R, Juvela S, et al. Natural history of brain arteriovenous malformations: a long-term follow-up study of risk of hemorrhage in 238 patients. Neurosurgery. 2008;63(5):823-829.

8. Kader A, Young WL, Pile-Spellman J, et al. The influence of hemodynamic and anatomic factors on hemorrhage from cerebral arteriovenous malformations. Neurosurgery. 1994;34(5):801-807.

9. BrunereauL, Gobin YP, Meder JF, et al. Intracranial duralarteriovenous fistulas with spinal venous drainage: relation between clinical presentation and angiographic findings. AJNR Am Neuro radiol. 1996;17(8):15491554.

10. Vanlandingham M, Fox B, Hoit D,et al. Endovascular treatment of intracranialduralarteriovenousfistulas. Neurosurgery. 2014;74(Suppl 1):S42-S49.

11. Hurst RW, Bagley LJ, Galetta S, et al. Dementia resulting from dural arteriovenous fistulas: the pathologic findings of venous hypertensive encephalopathy. AJNR Am J Neuroradiol. 1998;19(7):1267-1273.

12. Zeidman SM, Monsein LH, Arosarena O, et al. Reversibility of white matter changes and dementia after treatment of dural fistulas. AJNR Am J Neuroradiol. 1995;16(5):1080-1083.

13. Van Dijk JM, TerBrugge KG, Willinsky RA, Multi disciplinary management of spinal dural arteriovenous fistulas: clinical presentation and long-term follow-up in 49 patients. Stroke. 2012;33(6):1578-1583.

14. Lasjaunias P, Chiu M, ter Brugge K, et al. Neurological manifestations of intracranial dural arteriovenous malformations. $J$ Neurosurg. 1986;64(5):724-730.

15. Cognard C, Casasco A, Toevi M, et al. Dural arteriovenous fistulas as a cause of intracranial hypertension due to impairment of cranial venous outflow. J Neurol Neurosurg Psychiatry. 1998;65(3):308-316.

16. Tomlinson FH, Rufenacht DA, Sundt TM, et al. Arteriovenous fistulas of the brain and the spinal cord. J Neurosurg . 1993;79(1):16-27.

17. Signorelli F, McLaughlin N, Bojanowski MW. superficial siderosisas a manifestation of a dural arteriovenous fistula. Can J NeurolSci. 2011;38(2):367-369.

18. Cognard C, Gobin YP, Pierot L, et al. Cerebral dural arteriovenous fistulas: clinical and angiographic correlation with a revised classification of venous drainage. Radiology. 1995;194(3):671-680.

19. Borden JA, Wu JK, Shucart WA. A proposedclassification for spinal and cranial dural arteriovenous fistulous malformations and implications for treatment. J Neurosurg. 1995;82(2):166-179.

20. Gross BA, Du R. Natural history of cerebral arteriovenous malformations: a meta-analysis. J Neurosurg.2013;118(2):437-443.

21. Youssef PP, Schuette AJ, Cawley CM, et al. Advances in surgical approaches to dural fistulas. Neurosurgery. 2014;74 (Suppl 1):S32-S41.

22. Willinsky R, Goyal M, terBrugge K, et al. Tortuous, engorgedpialveinsin intracranial dural arteriovenous fistulas: correlations with presentation, location, and MR findings in 122 patients. AJNR Am J Neuroradiol. 1999;20(6):1031-1036.

23. Kawaguchi S, Sakaki T, Morimoto T, et al. Surgery for duralarteriovenous fistula in superior sagittal sinus and transverse sigmoid sinus. J Clin Neurosci. 2000;7(suppl1):47-49.

24. Gandhi D, Chen J, Pearl M, et al. Intracranial Dural ArteriovenousFistulas: Classification, Imaging Findings, and Treatment. AJNR Am JNeuroradiol. 2012;33(6):1007-1013.

25. Brunereau L, Gobin YP, Meder JF, et al. Intracranial dural arteriovenous fistulas with spinal venous drainage: relation between clinical presentation and angiographic findings. AJNR Am J Neuroradiol. 1996;17(8):1549-1554. 
26. da Costa L, Wallace MC, Ter Brugge KG, et al. The natural history and predictive features of hemorrhage from brain arteriovenous malformations. Stroke. 2009;40(1):100-105.

27. Guedin P, Gaillard S, Boulin A, et al. Therapeutic management of intracranial dural arteriovenous shunts with leptomeningeal venous drainage: report of 53 consecutive patients with emphasis on trans arterial embolization with acrylic glue. J Neurosurg. 2010;112(3):603-610.
28. Lv X, Li Y, Jiang C, et al. Endovascular treatment of brain arteriovenous fistulas. AJNR Am J Neuroradiol. 2009;30(4):851-856.

29. Pan HC, Sun MH, Sheehan J, et al. Radio surgery for dural carotidcavernous sinus fistulas: Gamma Knife compared with XKnife radio surgery. J Neurosurg. 2010;113(Suppl):9-20. 